\title{
Ironing out the Iron Requirements of Children and Adolescents
}

\author{
Raghu Pullakhandam, Bharati Kulkarni and Hemalatha Rajkumar \\ National Institute of Nutrition, Hyderabad, India \\ Correspondence to: Dr Bharati Kulkarni, Clinical Division, National Institute of Nutrition, Hyderabad, India. \\ dr.bharatikulkarni@gmail.com
}

I ron deficiency is one of the most prevalent and important micronutrient deficiencies worldwide, and iron deficiency anemia adversely impacts human capital in developing countries. Although, there is recent evidence suggesting that dietary iron intake and anemia may not be strongly related [1], iron fortification of staples has been one of the major strategies to tackle iron deficiency anemia worldwide. Supplemental iron, however, is a double-edged sword as iron intake exceeding requirement is known to cause multiple adverse effects, including oxidative stress, inflammation and modulation of gut microbiota $[2,3]$. Careful estimation of iron requirements in different physiological groups using rigorous methods is, therefore, extremely important.

Optimal growth during childhood and adolescence, and sustained health throughout life require multiple nutrients at specific levels; and these requirements vary across the life stages. Nutritionally demanding physiological states such as growth during childhood and adolescence, pregnancy and lactation impose additional requirements. Moreover, the nutrient absorption and metabolism in different population vary due to multiple reasons, including differences in the diet quality and bioavailability, body composition, and genetic makeup [4]. Estimating the nutrient requirements of population subgroups using population-specific data to the extent possible is of paramount importance.

The dietary recommendations serve as an important tool while formulating public health policies as these recommendations are used for evaluating the inadequacy of dietary intakes and help regulatory recommendations for fortification programs. The current nutrient requirements (RDA 2010) by Indian Council of Medical Research (ICMR) [5] provide a single value as the recommended dietary allowance (RDA) for each nutrient, which is more relevant for assessment of dietary adequacy for individuals rather than for population as a whole. Further, international framework of dietary reference intakes (DRIs) requires defining estimated average requirements (EAR) and RDAs of nutrients. The study published in this issue of Indian Pediatrics by Ghosh, et al. [6] provides estimates of the iron requirements of children and adolescents (1-17 years) under the framework of global DRIs (United Nations University's Food and Nutrition Program, in collaboration FAO, WHO and UNICEF) [7], and makes an important contribution to knowledge in the area of iron nutrition.

Computation of dietary requirements in children and adolescents is especially complicated because, apart from the basal or obligatory requirements, additional growth related requirements (which vary as per the rate of growth in different age groups) need to be considered. Basal requirements are estimated based on the norm that if the absorption of a nutrient from diet equals its obligatory losses, an individual is able to maintain the nutrient balance. Therefore, the sum of nutrient losses (basal losses and additional menstrual losses in case of adolescent girls) plus additional growth related requirements, when adjusted for bioavailability, provides a realistic estimate of nutrient requirements. This is often referred to as factorial approach. Ideally, the data on these variables measured in the same population should be used for deriving the nutrient requirement estimates but such data are often not available. The authors of this study [6] have, therefore, used relevant published data to estimate basal iron losses, including menstrual losses in adolescent girls and additional growth related requirements (due to blood volume expansion, tissue iron and storage pool). The values have been adjusted for age-specific bioavailability, and all these factors and their respective variances have been summed using appropriate statistical tools for computing EAR and RDA (RDA defined as the $97.5^{\text {th }}$ percentile of the distribution of requirements) for children from different age groups. The reference body weights of children and adolescents were based on the WHO Child Growth Standards 
(directly or derived) unlike the $95^{\text {th }}$ percentile value from the National Nutrition Monitoring Bureau (NNMB) data as used by the ICMR RDA. Due to methodological differences, the EAR and RDA values calculated by the authors are lower than the ICMR RDA in all age groups, except the RDA values for adolescent girls that are higher than the ICMR RDA. The EAR value $(18.5 \mathrm{mg} / \mathrm{d})$ in adolescent girls suggested for fortification by authors is lower than the ICMR RDA ( $27 \mathrm{mg} / \mathrm{d})$ currently being used for fortification programs.

This work is significant in many ways. First, it attempts to harmonize the iron requirement recommendations with international framework of DRIs. Second, the EAR-based dietary inadequacy assessment suggested by the authors is more realistic from population perspective, and would provide much lower estimate of inadequacy compared to the current practice of using RDA-based cut-point. Third, since food fortification standards are anchored on requirements of adolescent girls, the EAR metric derived for adolescent girls in the present study has implications in revising the fortification standards in the country. This is especially important when adolescents are likely to receive supplemental iron from multiple sources, including weekly iron for late supplementation program as well as from multiple iron-fortified foods; and there is a plausible risk of total iron take crossing the tolerable upper limit $(40 \mathrm{mg}$ /day in children below 14 years and 45 $\mathrm{mg} /$ day above 14 years age) [8]. Moreover, due to high prevalence of undernutrition in the country, majority of the children and adolescents are likely to have body weights far lower than the reference weights, which further increases the risk of excessive iron intakes.

It is, however, important to note that these nutrient requirement calculations involve complex analyses, and the estimates rely on a number of assumptions that are imperfect. The problem is further compounded by lack of data on iron losses as well as body iron stores that are representative of Indian population.

Important knowledge gaps exist as the basal iron losses, including menstrual iron loss and body iron stores, are based on extremely limited number of studies carried out in western populations. Further refinement of the DRIs, therefore, needs focused research to evaluate the basal losses as well as body stores of iron during growth in representative Indian participants, which requires methodological advances. Moreover, studies estimating iron absorption with different meals, especially composite meals, in different age groups are needed as the bioavailability was found to be major contributing factor to the higher EARs of iron for Indians compared to Western population.

Funding: None; Competing interest: None stated.

\section{REFERENCES}

1. Swaminathan S, Ghosh S, Varghese JS, Sachdev HS, Kurpad AV, Thomas T. Dietary iron intake and anemia are weakly associated, limiting effective iron fortification strategies in India J Nutr. 2019;149:831-39.

2. Moen IW, Bergholdt HKM, Mandrup-Poulsen T, Nordestgaard BG, Ellervik C. Increased plasma ferritin concentration and low-grade inflammation - a Mendelian randomization study. Clin Chem. 2018;64:374-85.

3. Paganini D, Zimmermann MB. The effects of iron fortification and supplementation on the gut microbiome and diarrhea in infants and children: A review. Am J Clin Nutr. 2017;106:1688s-93s.

4. Reddy VS, Palika R, Ismail A, Pullakhandam R, Reddy GB. Nutrigenomics: opportunities and challenges for public health nutrition. Indian J Med Res. 2018;148: 632-41.

5. Indian Council of Medical Research. Nutrient Requirements and Recommended Dietary Allowances for Indians. A Report of the Expert Group of the Indian Council of Medical Research. Hyderabad: National Institute of Nutrition, 2010.

6. Ghosh S, Sinha S, Shivakumar N, Thomas T, Sachdev HP, Kurpad AV. Daily iron requirements in healthy Indian children and adolescents. Indian Pediatr. 2019;56:551-5.

7. King JC, Garza C. Harmonization of nutrient intake values. Food Nutr Bull. 2007;28:S3-12.

8. Institute of Medicine (US) Panel on Micronutrients. Dietary Reference Intakes for Vitamin A, Vitamin K, Arsenic, Boron, Chromium, Copper, Iodine, Iron, Manganese, Molybdenum, Nickel, Silicon, Vanadium, and Zinc. Washington (DC): National Academies Press (US); 2001. 